\title{
A fast full-wave solver for calculating ultrasound propagation in the body
}

\author{
S.R. Haqshenas ${ }^{\mathrm{a}, \mathrm{b}, *}$, P. Gélat ${ }^{\mathrm{a}}$, E. van 't Wout $^{\mathrm{c}}$, T. Betcke ${ }^{\mathrm{b}}$, N. Saffari ${ }^{\mathrm{a}}$ \\ ${ }^{a}$ Department of Mechanical Engineering, University College London, London WC1E 7JE, UK \\ ${ }^{\mathrm{b}}$ Department of Mathematics, University College London, London WC1H OAY, UK \\ ${ }^{\mathrm{c}}$ Institute for Mathematical and Computational Engineering, School of Engineering and Faculty of Mathematics, Pontificia Universidad Católica de Chile, Santiago, Chile
}

\section{A R T I C L E I N F O}

\section{Keywords:}

Multiple-domain boundary element method

Helmholtz transmission problem

OSRC preconditioner

High intensity focused ultrasound

Cancer treatment planning

\begin{abstract}
A B S T R A C T
Therapeutic ultrasound is a promising non-invasive method for inducing various beneficial biological effects in the human body. In cancer treatment applications, high-power ultrasound is focused at a target tissue volume to ablate the malignant tumour. The success of the procedure depends on the ability to accurately focus ultrasound and destroy the target tissue volume through coagulative necrosis whilst preserving the surrounding healthy tissue. Patient-specific treatment planning strategies are therefore being developed to increase the efficacy of such therapies, while reducing any damage to healthy tissue. These strategies require to use high-performance computing methods to solve ultrasound wave propagation in the body quickly and accurately. For realistic clinical scenarios, all numerical methods which employ volumetric meshes require several hours or days to solve the full-wave propagation on a computer cluster. The boundary element method (BEM) is an efficient approach for modelling the wave field because only the boundaries of the hard and soft tissue regions require discretisation. This paper presents a multiple-domain BEM formulation with a novel preconditioner for solving the Helmholtz transmission problem (HTP). This new formulation is efficient at high-frequencies and where highcontrast materials are present. Numerical experiments are performed to solve the HTP in multiple domains comprising: (i) human ribs, an idealised abdominal fat layer and liver tissue, (ii) a human kidney with a perinephric fat layer, exposed to the acoustic field generated by a high-intensity focused ultrasound (HIFU) array transducer. The time required to solve the equations associated with these problems on a single workstation is of the order of minutes. These results demonstrate the great potential of this new BEM formulation for accurately and quickly solving ultrasound wave propagation problems in large anatomical domains which is essential for developing treatment planning strategies.
\end{abstract}

\section{Introduction}

High-intensity focused ultrasound (HIFU) therapy is a procedure which uses high-intensity ultrasound to thermally ablate a localised region of diseased tissue, leaving the surrounding healthy tissue intact. A successful clinical outcome requires a patient-specific HIFU treatment plan. The plan must be achieved prior to the intervention quickly and accurately. Excitation protocols of a multi-element transducer may then be determined [1], thus ensuring that diseased tissue is accurately ablated. This is usually achieved by recursively solving for the wave field in the body (i.e. the forward problem) using a linear full-wave model Gélat et al. $[1,2]$. The use of a linear full-wave model is physically justified as nonlinear propagation of ultrasonic waves is mainly confined to the focal region of the field [3]. This linear field (focusing vector) can then serve as input data into a nonlinear model so that precise thermal dose metrics may be accurately determined.
To date, several approaches have been used to model acoustic pressure fields for HIFU treatment planning applications. Modified Rayleigh-Sommerfeld integral methods, where the propagating medium is decomposed into multi-layered media, have been the subject of various studies [4-8]. This class of simulation method has a relatively low implementation effort and does not require the use of dense computational meshes. However, whilst Modified Rayleigh-Sommerfeld integral methods accounts for refraction during the transmission of an acoustic wave through a layered structure, they do not capture multiple reflections and cannot predict scattering resulting from step changes in tissue properties. The hybrid angular spectrum approach (HAS) $[9,10]$ extends the angular spectrum method to provide a computationally efficient alternative to full-wave ultrasound simulation methods, allowing it to cope with more complex geometries than modified Rayleigh-Sommerfeld methods [9]. However, HAS does not resolve the fullwave physics of multiple scattering and internal reflections within each

\footnotetext{
* Corresponding author at: Department of Mechanical Engineering, University College London, London WC1E 7JE, UK.

E-mail address: s.haqshenas@ucl.ac.uk (S.R. Haqshenas).
} 
medium $[6,10]$. Such deficiencies can be addressed using full-wave models such as finite-difference time domain (FDTD) approaches [11-13], and $k$-space pseudospectral methods $[5,14,15]$. In 3D however, these methods require the use of very dense volumetric grids to counteract the accumulation of numerical dispersion [5]. For example, in recent transcranial simulations, 12 grid points per wavelength were used [12]. $k$-space methods have been shown to require less dense computational grids than FDTD methods to provide the same level of accuracy [16]. Nevertheless, substantial computational challenges remain in trans-abdominal HIFU due to the large domain dimensions (typically $20 \times 20 \times 20 \mathrm{~cm}^{3}$ ), and the comparatively small wavelengths involved (around $1.5 \mathrm{~mm}$ in soft tissue at $1 \mathrm{MHz}$ ). The use of Cartesian grids as well as perfectly matched layers used in FDTD and $k$-space pseudospectral methods also lead to numerical artifacts, the wellknown staircasing effect and spurious reflections from the boundary of the computational domain, respectively.

In view of reducing the computational overheads, hybrid and mixed domains have formed the basis of some studies. A hybrid method including the Rayleigh-Sommerfeld integral, $k$-space pseudospectral method, and angular spectrum method was proposed [17]. This method forgoes a volumetric grid over the whole of the computational domain, confining the former to the heterogeneous region (i.e. in the vicinity of the ribs). A mixed domain method using an implicit analytical solution was described, which approximates the forward propagation of nonlinear waves in weakly heterogeneous media [18]. Whilst potentially more computationally efficient than their full-wave counterparts, the above approaches are effectively one-way propagation models and compromise the wave physics at the interfaces of localised heterogeneities. Additionally, the method proposed by Gu and Jing [18] precludes scenarios with high contrast scatterers such as bone.

The most promising full-wave computationally efficient 3D numerical method for HIFU treatment planning of transcostal tumours is currently the boundary element method (BEM) $[19,20]$. In BEM, the partial differential equation that models the wave propagation is essentially reformulated into a boundary integral equation defined on the interfaces. The boundary solutions and potential integrals are used to calculate the field at any point in the domain. Being based on Green's function representations, BEM is almost devoid of the numerical dispersion and dissipation effects commonly associated with numerical schemes such as $k$-space pseudospectral and finite-difference time domain methods $[21,22]$. For sound-hard scatterers, issues of long computing times have been overcome through analytical preconditioners used in conjunction with hierarchical matrix ( $\mathcal{H}$-matrix) compression techniques [20].

In order for such schemes to be incorporated into a clinically-relevant treatment planning framework, it is vital that they are extended to include the ability to deal with some degree of tissue heterogeneity. Whilst soft tissue such as skin, muscle and fat have similar compressional wave speeds and densities [23], fat attenuates ultrasound energy significantly and causes beam aberration at the focus. This impedes focal heating during HIFU treatment of renal cancer, due to the presence of perinephric fat $[15,24,25]$. It also remains to be established to which extent ultrasound can penetrate rib bone and how important it is to account for this phenomenon when modelling the propagation of ultrasound in the abdominal region. The ability to model the propagation of ultrasound through ribs and assess the importance of this relative to the case of locally reacting ribs [2] would be beneficial, particularly when studying the heat transfer mechanisms which lead to skin burns in HIFU patients [26]. There is therefore a requirement for resorting to multi-domain BEM to deal with the treatment planning complexities outlined above.

A number of multi-domain acoustic BEM formulations have been proposed [27-29]. In these approaches, the boundary integral equation (BIE) was applied to each domain. Due to the matrices resulting from domain discretisation being fully populated, the set-up time and memory consumption scale quadratically with respect to the number of degrees of freedom [30]. Fast BEM schemes have been introduced for the Helmholtz equation, resulting in a quasilinear complexity. The merits of such methods, namely the fast multipole method and hierarchical matrices, were discussed by Brunner et al. [30] where a scattering problem involving a $22 \mathrm{~m}$ long, $2 \mathrm{~m}$ thick cylinder immersed in water was analyzed for excitation frequencies up to $200 \mathrm{~Hz}$. Such fast BEM schemes have been applied to multi-domain Helmholtz problems. A fast multipole BEM method for 3D multi-domain acoustic scattering problems was described by Wu et al. [31].

The above studies investigate cases where the $k a$ (where $k$ is the wavenumber and $a$ is the characteristic size of the domain) is substantially less than that encountered in HIFU applications. Determining which BEM scheme is better suited for solving multi-domain Helmholtz problems at frequencies relevant to HIFU problems remains an active area of research. Previously, a fast BEM model was developed to solve the scattering from a single and perfectly rigid domain $[19,20]$. In this paper, we present a new fast multiple-domain transmission BEM model to compute both the scattered and transmitted ultrasonic fields in softtissue and bone. This formulation employs a novel preconditioner which is developed for high-frequency high-contrast problems. This advanced preconditioning together with $\mathcal{H}$-matrix compression techniques enable the wave equation to be solved in large domain sizes relative to the wavelengths involved. Therefore, it allows for realistic simulations using anatomical data and provides insightful information about HIFU fields inside the body. Furthermore, the model is generic and can therefore be applied to therapeutic ultrasound scenarios not discussed in this paper.

The formulation is presented in Section 2. The equations were implemented and solved using the open source library Bempp [32]. The simulations were validated against analytical solutions, where available. Subsequently, the scattered field was calculated for the following computational domains: i) an idealised layer of abdominal fat, human ribs and liver tissue, and ii) a human kidney model and a perinephric fat layer, see Section 3. In both scenarios, the incident field is generated by a spherical section array transducer operating at the frequency of $1 \mathrm{MHz}$. Finally, the suitability and limitations of this BEM scheme for use in HIFU treatment planning are discussed.

\section{Model formulation}

In this paper, we consider the forward problem only and aim to develop a fast and efficient solver for linear ultrasonic wave propagation in a medium made of the exterior domain $\Omega^{0}$ and non-overlapping domains $\Omega^{j}, j=1, \ldots, n$ with different physical properties, i.e. density, speed of sound and attenuation coefficient. A single-trace formulation which involves one Dirichlet and one Neumann data at each point of each interface is used and implemented. As a result, this formulation holds true where only two domains are in contact with each other, thus not allowing for triple points.

\subsection{Wave equation}

The linear full-wave propagation is modelled by the Helmholtz system as follows

$\begin{cases}\Delta p^{j}(\mathbf{x})+k_{j}^{2} p^{j}(\mathbf{x})=0, & \text { for } \mathbf{x} \in \Omega^{j} \\ \Delta p_{s}(\mathbf{x})+k_{0}^{2} p_{s}(\mathbf{x})=0, & \text { for } \mathbf{x} \in \Omega^{0}\end{cases}$

with boundary conditions defined below

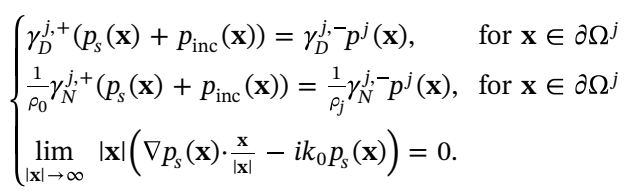

where $k_{j}, \rho_{j}, \gamma_{D}^{j, \pm}$ and $\gamma_{N}^{j, \pm}$ are the wavenumber, the mass density, and 


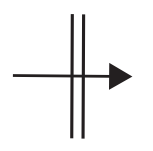

$p_{\text {inc }}$

$$
\Omega^{0}, k_{0}, \rho_{0}
$$
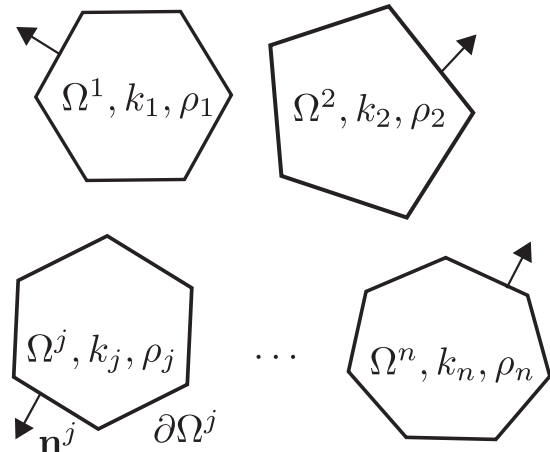

Fig. 1. Schematic diagram of the multiple domains. Refer to the text for the details.

the Dirichlet and Neumann traces associated with the domain $j$ for $j=1, \ldots, n$ taken from the exterior( + )/interior(-), respectively. The wavenumber $k_{j}$ is complex with the positive real part equal to $2 \pi / \lambda$ where $\lambda$ is the wavelength in meters, and the positive imaginary part being $\alpha_{0} f^{b}$ where $\alpha_{0}$ is the attenuation coefficient in neper $\mathrm{m}^{-1} \mathrm{~Hz}^{-\mathrm{b}}, f$ is the frequency in $\mathrm{Hz}$ and $b$ is the exponent. This frequency power law model is used to account for the attenuation of wave propagation in soft and hard tissues [33]. The traces are defined as follows:

$\left\{\begin{array}{l}\gamma_{D}^{j, \pm} p(\mathbf{x}):=\lim _{\Omega^{ \pm} \ni \mathbf{x}^{\prime} \rightarrow \mathbf{x}} p\left(\mathbf{x}^{\prime}\right) \\ \gamma_{N}^{j, \pm} p(\mathbf{x}):=\lim _{\Omega^{ \pm} \ni \mathbf{x}^{\prime} \rightarrow \mathbf{x}} \nabla p\left(\mathbf{x}^{\prime}\right) \cdot \mathbf{n}^{j}\left(\mathbf{x}^{\prime}\right)\end{array}\right.$

where $\mathbf{n}^{j}(\mathbf{x})$ is the normal vector on the boundary $\partial \Omega^{j}$ and points outwards to the exterior of domain $\Omega^{j}$ (for both $\gamma_{N}^{j, \pm}$ traces), see Fig. 1. Here, $p_{s}(\mathbf{x}), p_{\text {inc }}(\mathbf{x})$, are the scattered and incident pressure fields in the exterior, $p^{j}(\mathbf{x})$ is the pressure field in $\Omega^{j}$. For the sake of simplicity, the spatial variable $\mathbf{x}$ will be dropped from now on.

The last line in Eq. (2) is the Sommerfeld radiation condition that requires the scattered waves to be outgoing in the unbounded domain $\Omega^{0}$.

\subsection{Coupled boundary integral formulation}

The Calderón representation of the Helmholtz problem in the exterior domain $\Omega^{0}$ is given by, see Appendix A for the derivations,

$\left(\frac{1}{2} \mathrm{Id}-\mathrm{A}_{k_{0}}\right) \gamma^{+} p_{\text {tot }}=\gamma^{+} p_{s}$

where $\gamma^{+}:=\left[\gamma_{D}^{+} \gamma_{N}^{+}\right]^{T}$ is the exterior Cauchy trace operator at $\bigcup_{j=1}^{n} \partial \Omega^{j}$, Id is the identity operator, $A_{k_{0}}$ is the Calderón operator evaluated with the wavenumber of the exterior domain, $p_{\text {tot }}=p_{\text {inc }}+p_{s}$ is the total pressure in the exterior domain $\Omega^{0}$. Restricting the boundary integral operators to a single domain $j$ results in

$\left(\frac{1}{2} \mathrm{ld}-\mathrm{A}_{k_{0}}^{j}\right) \gamma^{j,+} p_{\text {tot }}-\sum_{i \neq j} \mathrm{~A}_{k_{0}}^{j i} \gamma^{i,+} p_{\text {tot }}$

$=\gamma^{j,+} p_{s}$.

Here, $\mathrm{A}_{k_{0}}^{j i}$ is the Calderón operator that takes the wavenumber $k_{0}$ and maps a surface potential at $\partial \Omega^{i}$ to $\partial \Omega^{j}$. The term $\sum_{i \neq j} \mathrm{~A}_{k_{0}}^{j i} \gamma^{i,+} p_{\text {tot }}$ accounts for all cross scatterings between domains $\Omega^{i}$ and $\Omega^{j}$ for $i=1, \ldots, n, i \neq j$.

For the interior of domain $\Omega^{j}$, the Calderon representation of the Helmholtz problem reads, see Appendix A for the derivations, $\left(\frac{1}{2} \mathrm{Id}+\mathrm{A}_{k_{j}}^{j}\right) \gamma^{j,-} p^{j}=\gamma^{j,-} p^{j}$,

where $\mathrm{A}_{k_{j}}^{j}$ is the Calderón operator evaluated in the interior of domain $\Omega^{j}$.

The boundary conditions shown in Eq. (2) can be represented as follows

$\gamma_{D}^{j,-} p^{j}=\gamma_{D}^{j,+} p_{\text {tot }}$,

$\gamma_{N}^{j,-} p^{j}=\frac{\rho_{j}}{\rho_{0}} \gamma_{N}^{j,+} p_{\text {tot }}$.

Defining $D_{j}:=\left[\begin{array}{cc}\text { Id } & 0 \\ 0 & \rho_{j} / \rho_{0}\end{array}\right]$, Eq. (7) transforms to $\gamma^{j,-} p^{j}=D_{j} \gamma^{j,+} p_{\text {tot }}$. Substituting this relationship into Eq. (6) and multiplying both sides with $D_{j}^{-1}$ produces

$\left(\frac{1}{2} \mathrm{ld}+\widetilde{\mathrm{A}}_{k_{j}}^{j}\right) \gamma^{j,+} p_{\text {tot }}=\gamma^{j,+} p_{\text {tot }}$,

where $\widetilde{\mathrm{A}}_{k_{j}}^{j}:=D_{j}^{-1} \mathrm{~A}_{k_{j}}^{j} D_{j}$.

Subtracting Eq. (5) from Eq. (8) produces

$\left(\mathrm{A}_{k_{0}}^{j}+\widetilde{\mathrm{A}}_{k_{j}}^{j}\right) \gamma^{j,+} p_{\text {tot }}+\sum_{i \neq j} \mathrm{~A}_{k_{0}}^{j i} \gamma^{i,+} p_{\text {tot }}$

$=\gamma^{j,+} p_{\text {tot }}-\gamma^{j,+} p_{s}$,

which rearranges to the final form of the formulation as follows

$\left(\mathrm{A}_{k_{0}}^{j}+\widetilde{\mathrm{A}}_{k_{j}}^{j}\right) \gamma^{j,+} p_{\text {tot }}+\sum_{i \neq j} \mathrm{~A}_{k_{0}}^{j i} \gamma^{i,+} p_{\text {tot }}$

$=\gamma^{j,+} p_{\text {inc }} \forall j=1, \ldots, n$.

This equation is called the Poggio-Miller-Chan-Harrington-Wu-Tsai (PMCHWT) formulation for the multiple-domain HTP. This represents a set of linear equations which can be condensed into a block system $\widehat{\mathrm{A}}_{k} \mathrm{u}=\mathrm{b}$ where

$\widehat{\mathrm{A}}_{k}:=\left[\widehat{\mathrm{A}}_{k}\right]_{j i}=\left\{\begin{array}{ll}\mathrm{A}_{k_{0}}^{j}+\widetilde{\mathrm{A}}_{k_{j}}^{j} & j=i \\ \mathrm{~A}_{k_{0}}^{j i} & j \neq i\end{array}\right.$,

and

$\mathrm{u}_{j}=\gamma^{j,+} p_{\text {tot }}, \quad \mathrm{b}_{j}=\gamma^{j,+} p_{\text {inc }}$.

In the above equations the Calderón operator is a $2 \times 2$ block matrix represented by

$\mathrm{A}_{k_{j}}^{j}:=\left[\begin{array}{cc}-\mathrm{K}^{j} & \mathrm{~V}^{j} \\ \mathbf{W}^{j} & \mathrm{~K}^{\prime j}\end{array}\right]$,

where $\mathrm{V}^{j}, \mathrm{~K}^{j}, \mathrm{~K}^{\prime j}, \mathrm{~W}^{j}$ are the single layer, double layer, adjoint double layer, and hypersingular boundary integral operators, respectively, see Appendix A for their definitions. Thus, to construct the PMCHWT formulation for the HTP for $n$ domains, i.e. to calculate Eq. (11), $4 n(n+1)$ boundary integral operators should be computed.

\subsection{Operator preconditioning}

The Galerkin method, also known as the method of moments, is used for the discretisation of boundary integral operators. The boundary $\partial \Omega^{j}$ is meshed by triangular elements. The discrete formulation results in a linear system of equations which corresponds to the matrix problem $\mathbf{A u}=\mathbf{b}$ where

$[\mathbf{A}]_{r q}=\widehat{\mathrm{A}}_{k} \eta_{q}, \eta_{r}, \quad \forall q, r=1,2, \ldots, z$ 
$\mathbf{u}=\left[u_{1}, u_{2}, \ldots, u_{z}\right]$ is the vector of unknown solution of the variational problem and $\mathbf{b}=\left[b_{1}, b_{2}, \ldots, b_{z}\right]$ with

$b_{r}=\gamma^{j,+} p_{\text {inc }}, \eta_{r}$.

Here, $z$ is the number of degrees of freedom (dofs), $\eta_{q}$ and $\eta_{r}$ are the trial and test functions, and .,. is the standard $L^{2}$ dual pairing. The test and trial functions are continuous piecewise linear functions on the triangular surface mesh. The reader is referred to [20,32] for further details.

This linear discrete system of equations is usually ill-conditioned at high driving frequencies and acoustic impedance mismatch between the exterior and interior domains. Consequently, iterative solvers such as GMRES converge very slowly. Hence, to improve the convergence rate of the iterative solvers, the preconditioned continuous problem $\mathrm{C}^{-1} \widehat{\mathrm{A}}_{k} \mathrm{u}=\mathrm{C}^{-1} \mathrm{~b}$ is solved instead where the operator $\mathrm{C}^{-1}$ is defined such that the spectral condition number of the preconditioned system is smaller than the original system. The operator preconditioners are designed using the mapping properties of the BIE operators - instead of the discretised matrix - which allows them to be easily combined with acceleration and compression algorithms [34,35].

The performance of conventional operator preconditioners of PMCHWT formulation substantially deteriorates in scenarios with large wavenumbers [36] and high contrast domains [37]. Such scenarios are common in HIFU treatment planning applications. Therefore, a new block-diagonal preconditioner was developed to regularise the PMCHWT equations for the multiple-domain Helmholtz transmission problem. This new preconditioner is based on the Neumann-to-Dirichlet, denoted by $\Lambda_{\mathrm{NtD}}$, and Dirichlet-to-Neumann maps, $\Lambda_{\mathrm{DtN}}$. $\Lambda_{\mathrm{NtD}}$ maps the normal gradient of the pressure field on the boundary $\partial \Omega^{j}$ to the pressure field on the surface. $\Lambda_{\text {DtN }}$ performs the inverse map. These maps evaluated in the exterior domain satisfy the following relationships $[38,39]$,

$\Lambda_{\mathrm{DtN}}^{j,+} \bigvee^{j,+}=-\frac{1}{2} \mathrm{I}+\mathrm{K}^{j,+}$,

$\Lambda_{\mathrm{NtD}}^{j,+} \mathrm{W}^{j,+}=-\frac{1}{2} \mathrm{I}-\mathrm{K}^{j,+}$.

The double layer operator $\left(\mathrm{K}^{j,+}\right)$ and adjoint double layer operator $\left(K^{j,+}\right)$ are compact on sufficiently smooth surfaces and therefore $\Lambda_{\mathrm{DtN}}^{j,+} \mathrm{V}^{j,+}$ and $\Lambda_{\mathrm{NtD}}^{j,+} \mathrm{W}^{j,+}$ are better conditioned than single layer and hypersingular operators. Thus, the DtN and NtD maps are used to build the following block-diagonal preconditioner

$\mathrm{C}^{-1}:=[\mathrm{C}]_{j i}=\left\{\begin{array}{ll}\mathrm{C}^{j} & j=i \\ 0 & j \neq i\end{array}\right.$,

where

$\mathrm{C}^{j}:=\left[\begin{array}{ll}\Lambda_{\mathrm{DtN}}^{j,+} & \\ & \Lambda_{\mathrm{NtD}}^{j,+}\end{array}\right]$.

The above preconditioner is designed to be a block-diagonal matrix. This has two major advantages. First, this results in fast matrix-vector operations which scale linearly with the number of domains rather than quadratically for full matrices. Second, the preconditioner is highly accurate since it is based on a model for close interactions at the surface of the scatterers (diagonal blocks of $\widehat{A}_{k}$ ) which have a larger impact on the overall accuracy of the solution relative to distant interactions.

In order to use this preconditioner with the PMCHWT formulation, the Calderón operators should be vertically permuted to place the hypersingular and single layer operators on the main diagonal. To preserve the RHS of Eq. (10), the blocks of the unknown vector $\mathbf{u}_{j}=\left[\gamma_{D}^{j,+} p_{\text {tot }} \gamma_{N}^{j,+} p_{\text {tot }}\right]^{T}$ are permuted as well.

To implement this preconditioner, we need to calculate the DtN and NtD maps. These are non-local pseudo-differential operators with no closed-form solutions. However, an accurate approximation based on the high frequency asymptotics of them can be achieved using the onsurface radiation condition (OSRC) method $[40,41]$. The expressions of the $\mathrm{NtD}$ and $\mathrm{DtN}$ maps approximated by the OSRC approach are given by

$\widetilde{\Lambda_{\mathrm{DtN}}}=i k \sqrt{1+\frac{\Delta_{\Gamma}}{k_{\epsilon}^{2}}}$

$\widetilde{\Lambda_{\mathrm{NtD}}}=\frac{1}{i k}\left(1+\frac{\Delta_{\Gamma}}{k_{\epsilon}^{2}}\right)^{-1 / 2}$

where $k_{\epsilon}=k+i \in$ where $\epsilon>0$ is a damped wavenumber and $\Delta_{\Gamma}$ denotes the Laplace-Beltrami operator. The damping avoids any singularities in the operators and is chosen as $\epsilon=0.4 k^{-1 / 3} R_{\epsilon}^{-2 / 3}$ with $R_{\epsilon}$ the radius of the scatterer. This choice can be shown to be optimal for spherical objects and a good guideline for general geometries [42]. The square root operations in above equations are approximated with a Padé series expansion with $N_{\text {Pade }}$ terms. Each term is a surface Helmholtz equation with a different complex-valued wavenumber. The Galerkin discretisation of which results in a set of sparse linear systems that can efficiently be solved with sparse LU factorisation. Hence, the preconditioner requires sparse arithmetic only, which causes little overhead compared to the dense arithmetic for the model formulation. The reader is referred to $[19,20,39]$ for further details. Since the OSRC approximations of the NtD and DtN maps, Eqs. (19a) and (19b), are used to compute the elements of the preconditioner Eq. (17), it will be referred to as the OSRC block diagonal preconditioner.

\section{Numerical experiments}

The number of surface elements required by BEM to represent the wave propagation is of $O\left(k^{2}\right)$ which results in the memory footprint of $O\left(k^{4}\right)$, as Galerkin discretisation of the boundary integral operators results in dense matrices. This prohibits the implementation of this BEM formulation for HIFU treatment planning on a typical computational platform. To alleviate this problem, the hierarchical matrix compression with the tolerance set to $10^{-6}$ is used to assemble the discretised boundary integral operators in a compressed format. This technique is based on an adaptive cross approximation of boundary integral operators, the kernel of which is the Green's function (see Appendix A). The regularity of the Green's function increases with the distance between the source and observer. Consequently, the elements of the matrix that correspond to these distant interactions are more regular than short distance interactions and can hence be approximated with smooth functions. Recursively using these properties for groups of elements results in a low-rank approximation of blocks of the matrix. At high frequencies, classical compression often deteriorates and efficiency bounds scale poorly for $k \rightarrow \infty$, thus necessitating specialized directional compression techniques. On the contrary, computational benchmarks in Betcke et al. [20] confirm a significantly better performance than the asymptotic worst-case scenarios. In practice, excellent compression rates have been observed for the models considered here. Refer to van 't Wout et al. [19] and Betcke et al. [20] for complementary information about hierarchical matrices and their application for solving Helmholtz problems.

A workstation with 32 processors Intel $^{\oplus}$ Xeon(R) CPU E5-2683 
Table 1

Physical properties of the domains $[45,46]$.

\begin{tabular}{ccccc}
\hline domain & $\rho$ & $c$ & $\alpha_{0}$ & $b$ \\
& {$\left[\mathrm{~kg} \mathrm{~m}^{-3}\right]$} & {$\left[\mathrm{m} \mathrm{s}^{-1}\right]$} & {$\left[\mathrm{Np} \mathrm{m}^{-1}\right.$ at $\left.1 \mathrm{MHz}\right]$} & $\begin{array}{c}b \\
{[\mathrm{DL}]}\end{array}$ \\
\hline ribs & 1178 & 2117.5 & 47 & 1 \\
fat & 917 & 1412 & 9.3 & 1 \\
kidney & 1066 & 1554.3 & 2.8067 & 1 \\
liver & 1070 & 1640 & 7 & 1.30 \\
water & 1000 & 1500 & 0.015 & 2 \\
\hline
\end{tabular}

v4@2.10 GHz) and 512 GB RAM was used for performing the following numerical experiments. First, the preconditioned formulation and its implementation was validated against analytical solutions for a model problem consisting of a spherical object in Section 3.1. Subsequently, the scattered field from i) human ribs and an idealised abdominal fat layer, ii) a human kidney and a perinephric fat layer, exposed to the acoustic field generated by a HIFU array transducer is computed and discussed in Section 3.2.

The presented formulation was implemented in Python using the open source Bempp library [32], version 3.3.4. The GMRES algorithm of the SciPy library with the restart parameter set to 100 was used for solving the discrete system of equations. The GMRES scheme terminates when the relative residual is below a specified relative tolerance, which was set to $10^{-5}$. The efficiency of the OSRC preconditioner Eq. (19) is affected by parameters $R_{\epsilon}$ and $N_{\text {Pade }}$. Although one can choose different $R_{\epsilon}$ and $N_{\text {Pade }}$ for different domains, one set of values were selected to construct the preconditioner for the sake of simplicity. The larger $N_{\text {Pade }}$ the more accurate the approximation of DtN and NtD maps are, but at the cost of more numerical operations at each iteration. The numerical investigations demonstrate the following points: (i) a relatively small size of Padé series, 4 for spheres and 8 for anatomical meshes, is sufficient to calculate the OSRC preconditioner and achieve expected performance, (ii) setting $R_{\epsilon}$ to a value of one order of magnitude smaller than the characteristic size of the largest domain in the model leads to a fast convergence.

\subsection{Single sphere model}

The analytical solution for a penetrable acoustic sphere is available using the classical series expansion method $[43,44]$. This single sphere model has been extensively used in the literature as a standard model to validate and characterise the accuracy of numerical methods against analytical solutions. Likewise, in order to validate the new formulation presented in this paper and perform sensitivity analyses, the HTP problem of a single sphere object impinged by a plane wave is considered as the first numerical experiment. The driving frequency is assumed to be $1 \mathrm{MHz}$ which is typically used in HIFU applications for the liver and kidney. The exterior medium is assumed to be water and the sphere possesses the physical properties of (i) rib bones and (ii) abdominal fat. The physical properties are listed in Table 1 . The diameter of the sphere is $1 \mathrm{~cm}$ which is large enough to include multiple interior reflections at the wavelength of the sphere material, and which is of the order of magnitude of the anatomical heterogeneities considered in Section 3.2 of this paper. The problem was solved for a range of mesh densities, the latter parameter corresponding to the number of elements per wavelength associated with the material which has the smallest speed of sound. The value of $R_{\epsilon}=5 \times 10^{-4} \mathrm{~m}$ is used for all the sphere simulations.

In order to compare BEM and analytical results, the $l_{2}-$ norm of the

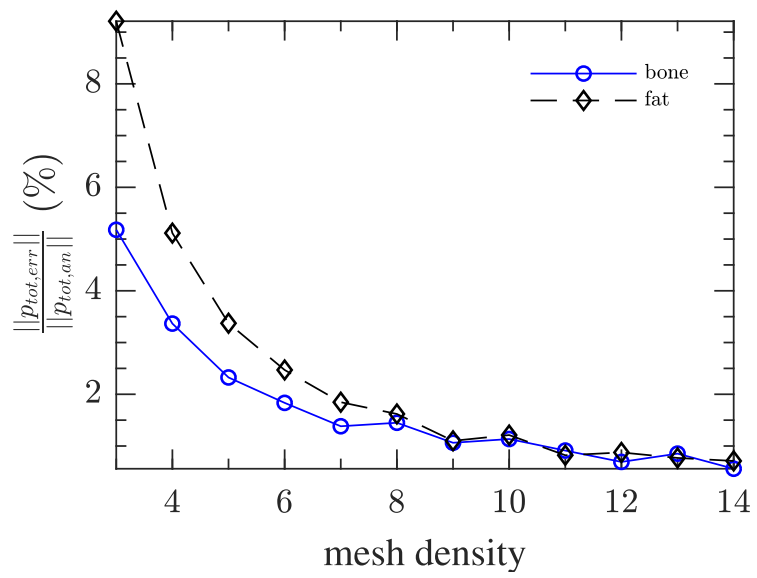

Fig. 2. The percentage of the $l_{2}-$ norm of the difference between the computed total pressure by BEM and the analytical total pressure at different mesh densities. The single sphere model is used.

difference in the total pressure, $p_{\text {tot,err }}=p_{\text {tot }}-p_{\text {tot,an }}$ was calculated where $p_{\text {tot }}$ is the computed pressure in the field by the BEM formulation and $p_{\text {tot,an }}$ is the analytical total pressure. The computational grid is a uniform $200 \times 200$ grid spanning from $-3 R$ to $3 R$, where $R$ is the radius of the sphere, through the centre of the sphere. The predictions of the total field becomes more accurate, in the sense of $l_{2}$ - norm, as the mesh density increases, see Fig. 2. Nevertheless, with rather coarse surface meshes of 6 elements per wavelength, the error is below 3\%. This choice permits the prediction of the total field relatively accurately and fast- the computation time per iteration (denoted by $\Delta t$ ) is about $0.2 \mathrm{~s}$ for both fat and bone spheres, see Fig. $3 \mathrm{~b}$. Bone is a high contrast material with $Z_{\text {int }} / Z_{\text {ext }}=5.2$ where $Z_{\text {int }}$ and $Z_{\text {ext }}$ are the characteristic specific acoustic impedances of the interior and exterior domains. For such materials, making the surface mesh finer improves the convergence rate of the iterative solver, as shown in Fig. 3a. However, this comes at the expense of longer computation time per iteration because the matvec operations (the product of a matrix by a vector) of larger matrices need to be carried out. This improvement is negligible when the material contrast is small, i.e. fat with $Z_{\text {int }} / Z_{\text {ext }}=0.86$.

\subsection{Anatomical model problems}

There is currently significant interest in both the research and clinical communities in the use of HIFU for the thermal ablation of liver and renal tumours [47]. This modality has been deployed into the clinic and has been the subject of a number of clinical trials. Currently, treatment planning is non-existent to rudimentary, relying instead on MR thermometry for guidance. The latter modality suffers from uncertainties which are dependent on the tissue type being monitored. In view of developing treatment plans, we will consider these two anatomical problems in this section and apply the new formulation presented in this paper to solve the forward problem. The results presented here are calculated for an incident field generated by a HIFU array transducer. The radius of the curvature of the array is $18 \mathrm{~cm}$, the $\mathrm{f}$ number is 1.18 and the focal point is located at the global origin by convention. The array features a pseudo-random spatial distribution of 256 piston elements, each of $6 \mathrm{~mm}$ diameter and facing towards the focal point. The details of calculating the HIFU incident field are presented in previous works, e.g. see [19], and will not be repeated here. 


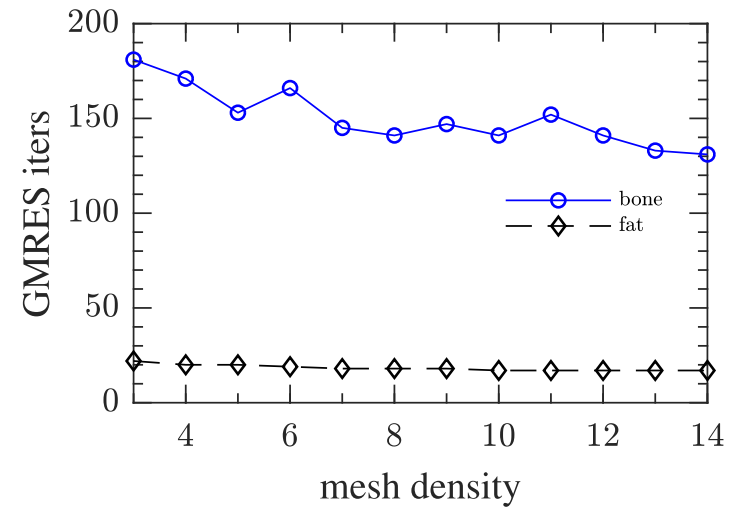

(a) Number of GMRES iterations at different mesh densities.

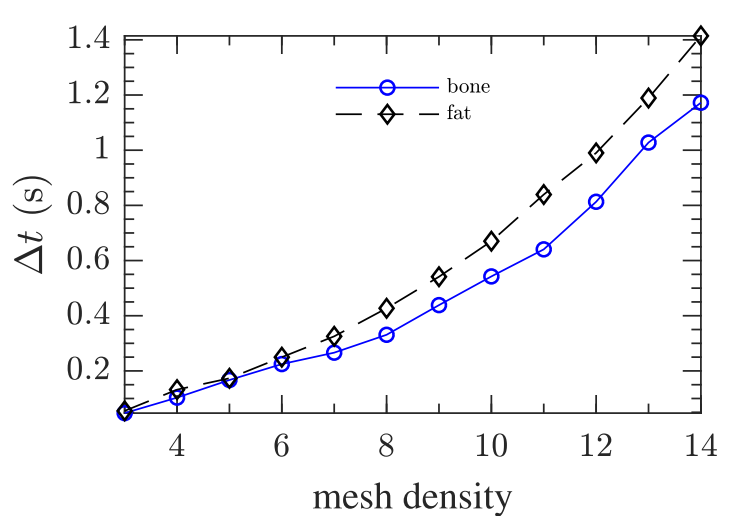

(b) The average GMRES time per iteration, i.e.

$\Delta t$, at different mesh densities.

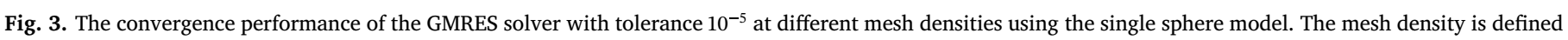
as the number of elements per wavelength.

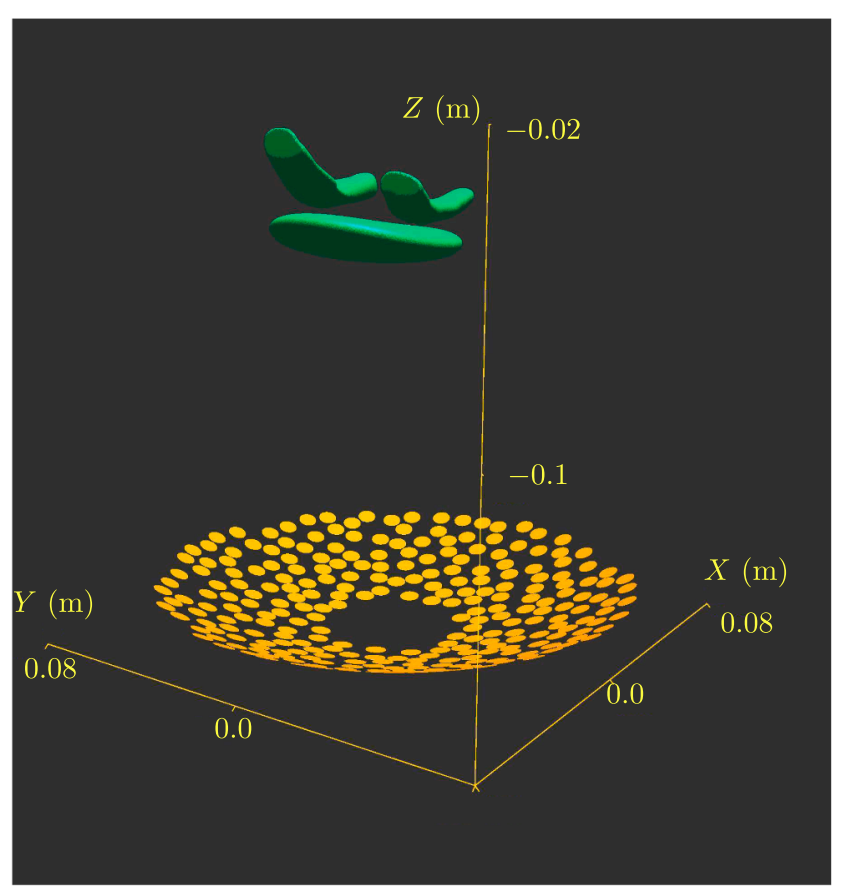

Fig. 4. Schematic diagrams of the HIFU array, the idealised abdominal fat layer and the ribcage, the intersection of visualisation axes is $[-0.08,-0.08,-0.18]$.

The first anatomical example involves a clinically relevant scenario of targeting a tumour of the liver located at an intercostal space $3.5 \mathrm{~cm}$ behind ribs 10 and 11, on the right side, see Fig. 4. Considering that the incident wave is a highly focused beam, the obstacles outside the beam width have negligible effect on the pressure at the focus and can therefore be eliminated to reduce the size of the problem. Hence, instead of using the model of the complete rib cage we retain only ribs 10 and 11 which are in the path of the HIFU beam. Therefore, the scattering objects (domains) are i) an idealised abdominal fat layer located in the vicinity of the anterior of the ribcage, and ii) two human ribs. These two domains are immersed in an infinite exterior domain possessing physical properties representative of the human liver. Ribs generally consist of a cancellous bone core enclosed by a shell of cortical bone, the thickness of which varies with gender and age. Examining micro CT scans of ribs, it is apparent that the majority of the volume of a rib consists of cancellous bone $[48,49]$. Thus, the physical properties of ribs used in the numerical simulations in this paper correspond to those of cancellous bone. The acoustic properties of all tissue media are listed in Table 1 . The fat layer was assumed to possess an ellipsoidal cross section with a thickness of $10 \mathrm{~mm}$ close to the axis of the transducer. The distance between the surface of the fat layer and the ribs vary from $1 \mathrm{~mm}$ to $15 \mathrm{~mm}$.

The surface meshes of domain $j$ are made of triangles with an average element size of $\lambda / 4$ where $\lambda=\min \left(\lambda_{0}, \lambda_{j}\right), j=1,2$. This resulted in (i) 44924 nodes $(89,848 \mathrm{dofs})$ in the fat domain, and (ii) 30088 nodes $(60,176 \mathrm{dofs})$ in the ribs domain.

In the first instance, a simulation was carried out omitting the fat layer. The GMRES solver (with the same tolerance and restarts as before) solved the preconditioned equations for the ribs model in 74 iterations and $2 \mathrm{~min}$. Adding the fat layer to the model increased the number of iterations to 82 and the runtime to just under $9 \mathrm{~min}$. Here, $R_{\epsilon}=10^{-4} \mathrm{~m}$ was used to calculate the OSRC preconditioner, Eq. (19). The computation of the discrete matrices with the standard $\mathcal{H}$-matrix compression technique took 80 and $465 \mathrm{~min}$ for the ribs only and fat and ribs problems, respectively. The simulation results are depicted in Figs. 5 and 6. It can be observed that the presence of the fat layer leads to a drop in the peak pressure magnitude at the focus. Another interesting observation is the formation of a pre-focal high-pressure area caused by the constructive interference of the diffracted and transmitted waves through the fat layer. The significance of this observation and its clinical ramifications will however depend on the size, geometry and location of the fat layer with respect to the tumour and other tissue heterogeneities. The patient specificity of such features reinforces the need for treatment planning using validated numerical models, prior to a HIFU intervention, so that the potential impact of pre-focal heating may be gauged and subsequently mitigated. Figs. $7 \mathrm{a}$ and $\mathrm{b}$ show the axial and lateral waveforms through the focus (i.e. along the $Z$ and $Y$ 


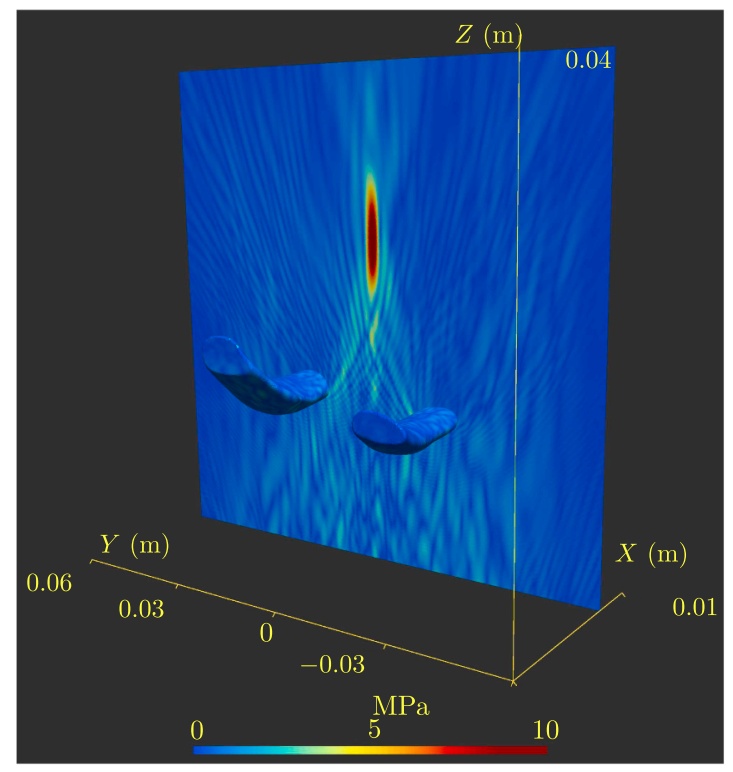

(a) Two ribs without a fat layer.

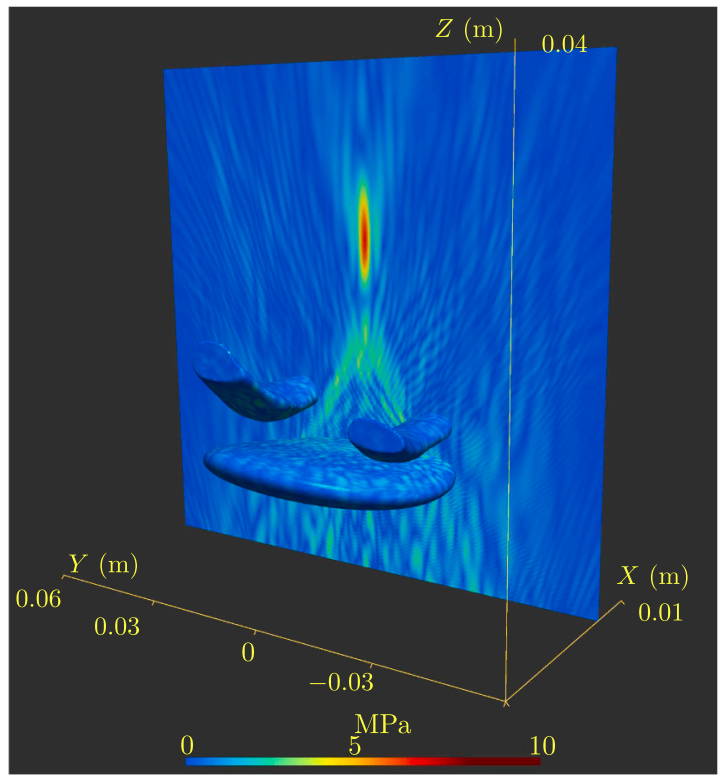

(b) Two ribs and a fat layer.

Fig. 5. Calculated absolute value of $p_{\text {tot }}$ for the case of two ribs (a) without a fat layer and (b) with a fat layer.

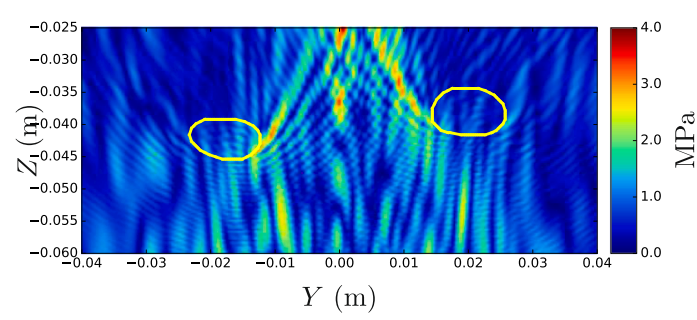

(a) Two ribs without a fat layer.

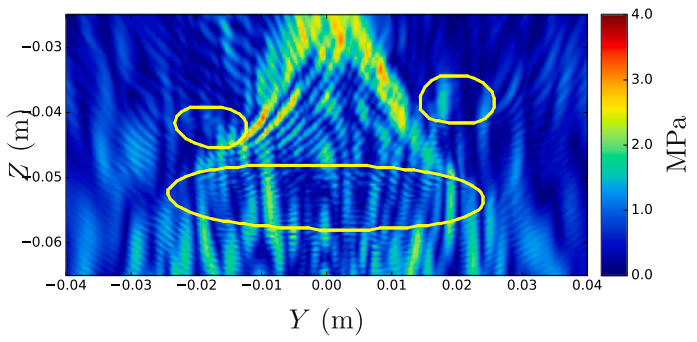

(b) Two ribs and a fat layer.

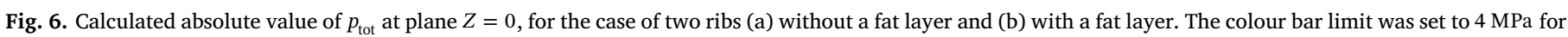
a better visualisation of the interior fields. Contours specify the boundary of the ribs and the fat layer.

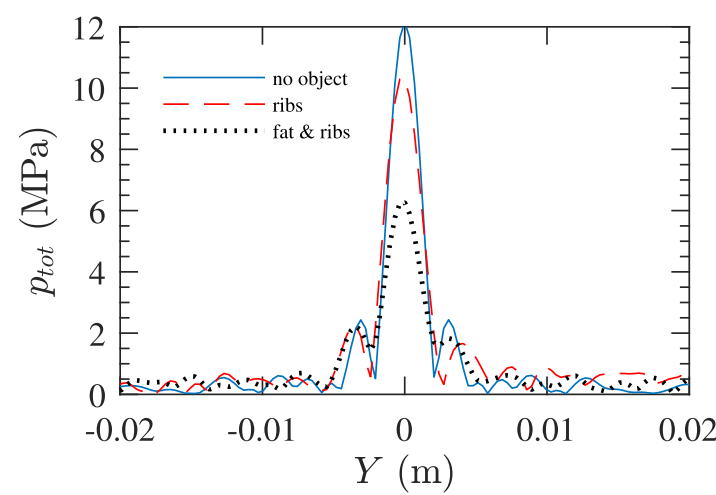

(a)

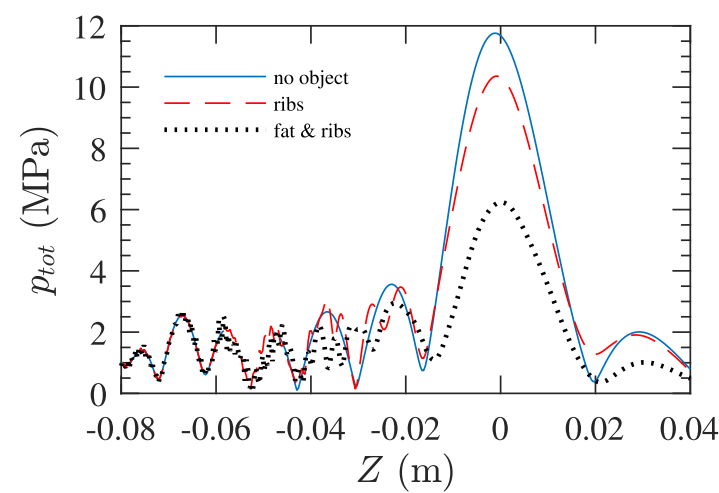

(b)

Fig. 7. The absolute value of $p_{\text {tot }}$ along (a) $Y$ axis at $X=0, Z=0$, (b) along $Z$ axis at $X=0, Y=0$. 


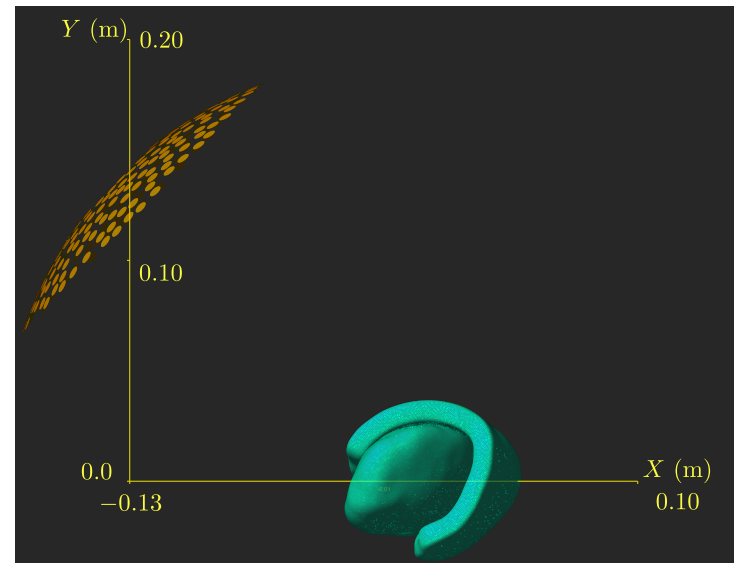

Fig. 8. Schematic diagrams of the HIFU array, perinephric fat layer and the kidney, the intersection of visualisation axes is $[-0.13,0,0]$

axes), respectively. The peak pressure at focus is reduced by almost $15 \%$ when accounting for transmission and scattering by ribs. Augmenting the model by including the fat layer, the peak pressure at focus decreases by about $50 \%$.

The second anatomical example involves another clinically relevant scenario using HIFU to ablate a tumour of the kidney. The apex of the spherical section array is positioned at $[-0.13,0.13,0] \mathrm{m}$ and the axis of transducer is colinear with the $[1,-1,0]$ vector, thus resulting in the geometric focus of the array being inside the kidney, see Fig. 8. The scattering domains are (i) a perinephric fat layer enclosing the kidney, and (ii) a human kidney model. These two domains are immersed in an infinite exterior domain possessing physical properties of water. The physical properties of all domains are shown in Table 1 . The thickness of the perinephric fat layer is about $10 \mathrm{~mm}$ and is placed $1 \mathrm{~mm}$ away from the kidney (constant gap).

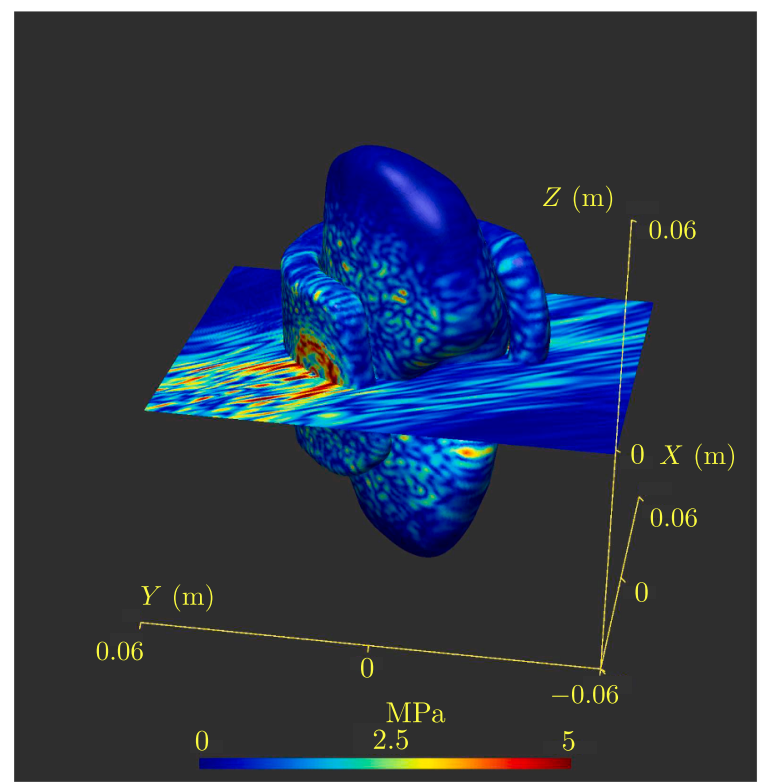

(a)

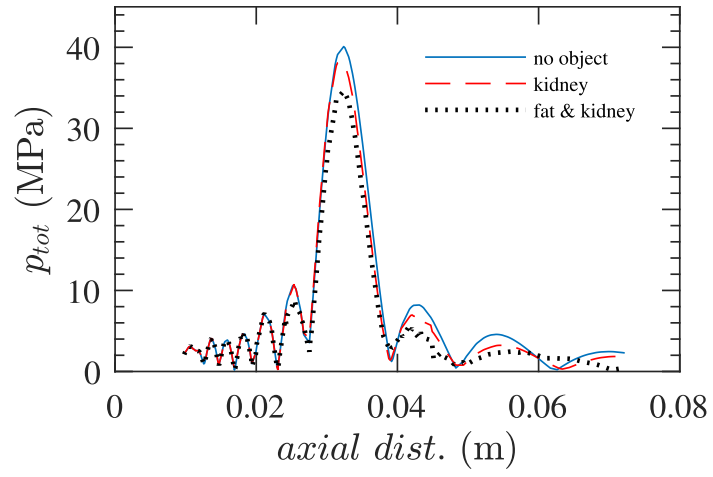

Fig. 10. The absolute value of $p_{\text {tot }}$ at $Z=0$ plane along the axis of propagation, i.e. $[1,-1,0]$ vector. The axial distances are determined from the centre of the array located at.$[-0.13,-0.13,0]$.

Similar to the previous examples, the triangular surface meshes have an average element size of $\lambda / 4$. The fat and kidney domains have 47,766 and 65,870 nodes (95,532 and 131,740 dofs), respectively. The $R_{\epsilon}$ parameter of the OSRC preconditioner was set to $10^{-3} \mathrm{~m}$. The preconditioned equations were solved in 69 iterations which took about 35 min. Simulations are shown in Figs. 9a and b. Considering that the material contrasts in both domains are small, i.e. $Z_{\text {fat }} / Z_{\text {water }}=0.86$ and $Z_{\text {kidney }} / Z_{\text {water }}=1.10$, the aberration of the HIFU beam is negligible. This is better displayed in Fig. 10 which shows the magnitude of the axial pressure for different scenarios. The peak pressure at the focus is reduced by $15 \%$ due to the presence of the perinephric fat layer and the kidney. Eliminating the perinephric fat layer and keeping the kidney, the peak pressure at the focus only reduces by $5 \%$. This problem was solved in 45 iterations and $12 \mathrm{~min}$. The computation time to discretise and populate the matrix with $\mathcal{H}$-matrix compression is about 20 and $40 \mathrm{~h}$ for the kidney only and kidney and perinephric fat models, respectively.

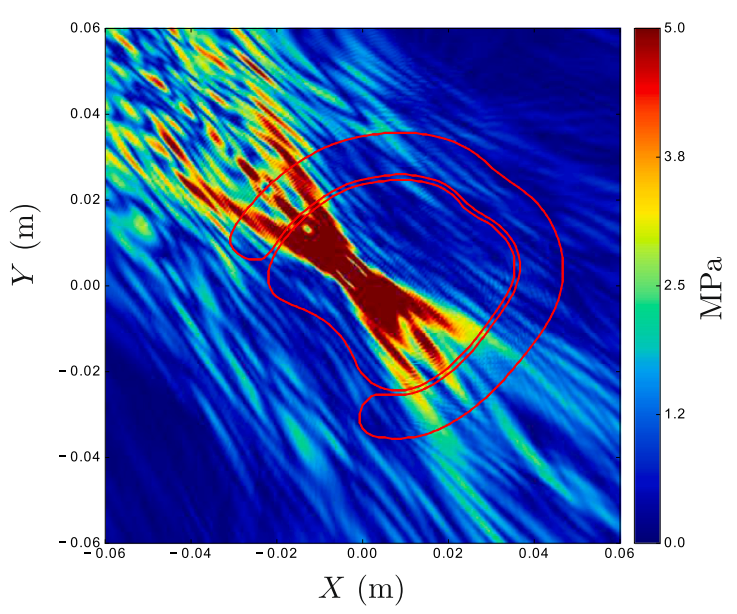

(b)

Fig. 9. Calculated absolute value of $p_{\text {tot }}$ for the case of a perinephric fat layer and the kidney: (a) in 3D, (b) at $Z=0$ plane. 


\section{Conclusion}

This paper presents an innovative fast multiple-domain BEM formulation for solving the Helmholtz transmission problem. The formulation uses a variation of the multiple-domain PMCHWT equation and a novel OSRC preconditioner. The numerical experiments on a single workstation demonstrated the great performance of this new formulation for solving the Helmholtz transmission problem at high $\mathrm{ka}$ values (i.e. high frequencies and/or large - relative to the wavelength computational domains) and where there is high variation in material properties. In such scenarios, the HTP becomes ill-conditioned and computationally intractable. The numerical methods which use volumetric meshes face challenges to solve those problems on a workstation, demanding access to computer clusters. Nonetheless, we have shown that the new OSRC preconditioner and the multiple-domain BEM formulation deliver an efficient solution for modelling linear ultrasound propagation in large computational domains consisting of soft and hard tissues.

These advantages of the developed formulation make it stand out for HIFU treatment planning applications. Numerical simulations were performed using anatomical meshes and a HIFU array transducer designed for treating abdominal tumours. The pressure fields were calculated for (i) a model of a human ribcage and an idealised abdominal fat layer, and (ii) a human kidney and perinephric fat layer, exposed to the acoustic field generated by a 256-element HIFU array transducer at $1 \mathrm{MHz}$. These simulations provided important insightful observations as follows: the presence of tissue heterogeneities and strong scatterers such as bone can lead to substantial aberration of the focus, the formation of the pre-focal high-pressure area, and reduction in the peak pressure. The significance of these effects are patient specific which reinforces the need for treatment planning using validated numerical models prior to a HIFU intervention. Furthermore, these results promote the application of the new OSRC block diagonal preconditioner and the multiple-domain BEM algorithm for fast and accurate patientspecific HIFU treatment planning. Nevertheless, the computational performance of this solver can be further improved by speeding up the calculation of the Calderón operators. This is the subject of our current research. We are also incorporating the developed model into a software package for calculating ultrasound propagation in the body and cancer treatment planning.

\section{Declaration of Competing Interest}

The authors declare that they have no known competing financial interests or personal relationships that could have appeared to influence the work reported in this paper.

\section{Acknowledgement}

This work was supported by the EPSRC[Grant No. EP/P012434/1] and CONICYT [FONDECYT No. 11160462].

\section{Appendix A. Calderón operator}

Solving the Helmholtz transmission problem with the BEM requires to reformulate the Helmholtz equation in the volume to boundary integral equations at the interfaces of two domains. This can be achieved by the aid of representation formulae which determine the acoustic fields from surface potentials at the boundaries. The representation formula for the domain $j,(j \neq 0)$ reads

$p^{j}=\mathrm{SL}_{k_{j}}^{j} \psi^{j}\left(p^{j}\right)-\mathrm{DL}_{k_{j}}^{j} \phi^{j}\left(p^{j}\right)$,

where $\phi^{j}\left(p^{j}\right)$ and $\psi^{j}\left(p^{j}\right)$ are the jump potentials, and $\mathrm{SL}_{k_{j}}^{j}$ and $\mathrm{DL}_{k_{j}}^{j}$ are the single layer and double layer potential integral operators, respectively, and are given by

$\left[\mathrm{S}\left\llcorner^{j} \psi\left(p^{j}\right)\right](\mathbf{x})=\int_{\partial \Omega^{j}} G_{k_{j}}(\mathbf{x}, \mathbf{y}) \psi(\mathbf{y}) d \Gamma(\mathbf{y})\right.$

for $\mathbf{x} \notin \partial \Omega^{j}$,

$\left[\mathrm{DL} L^{j} \phi\left(p^{j}\right)\right](\mathbf{x})=\int_{\partial \Omega^{j}} \frac{\partial G_{k_{j}}(\mathbf{x}, \mathbf{y})}{\partial \mathbf{n}^{j}(\mathbf{y})} \phi(\mathbf{y}) d \Gamma(\mathbf{y})$

for $\mathbf{x} \notin \partial \Omega^{j}$,

where $G_{k_{j}}(\mathbf{x}, \mathbf{y})$ denotes the Green's function for the Helmholtz equation given by

$G_{k j}(\mathbf{x}, \mathbf{y})=\frac{e^{i k_{j}|\mathbf{x}-\mathbf{y}|}}{4 \pi|\mathbf{x}-\mathbf{y}|}$

for $\mathbf{x}, \mathbf{y} \in \Omega^{j}$ and $\mathbf{x} \neq \mathbf{y}$.

Taking the Cauchy trace, $\gamma^{j, \pm}:=\left[\begin{array}{ll}\gamma_{D}^{j, \pm} & \gamma_{N}^{j, \pm}\end{array}\right]^{T}$, of the representation formula Eq. (A.1) produces

$\gamma^{j, \pm} p^{j}=\gamma^{j, \pm} \operatorname{SL}_{k_{j}}^{j}\left(\psi^{j}\left(p^{j}\right)\right)-$

$\gamma^{j, \pm} \operatorname{DL}_{k_{j}}^{j}\left(\phi^{j}\left(p^{j}\right)\right)=$

$\left[\begin{array}{l}\gamma_{D}^{j, \pm} \operatorname{SL}_{k_{j}}^{j}\left(\psi^{j}\left(p^{j}\right)\right)-\gamma_{D}^{j, \pm} \operatorname{DL}_{k_{j}}^{j}\left(\phi^{j}\left(p^{j}\right)\right) \\ \gamma_{N}^{j, \pm} \operatorname{SL}_{k_{j}}^{j}\left(\psi^{j}\left(p^{j}\right)\right)-\gamma_{N}^{j, \pm} \operatorname{DL}_{k_{j}}^{j}\left(\phi^{j}\left(p^{j}\right)\right)\end{array}\right]$.

In order to further simplify this, we need to use the jump relations and their regularity across the boundary. The jump relations read 


$$
\begin{aligned}
\gamma_{D}^{j, \pm} \mathrm{SL}_{k_{j}}^{j}\left(\psi^{j}\left(p^{j}\right)\right) & =\mathrm{V}^{j} \psi^{j}\left(p^{j}\right), \\
\gamma_{D}^{j, \pm} \mathrm{DL}_{k_{j}}^{j}\left(\phi^{j}\left(p^{j}\right)\right) & =\mathrm{K}^{j} \phi^{j}\left(p^{j}\right) \pm \frac{1}{2} \phi^{j}\left(p^{j}\right), \\
\gamma_{N}^{j, \pm} \mathrm{SL}_{k_{j}}^{j}\left(\psi^{j}\left(p^{j}\right)\right) & =\mathrm{K}^{\prime j} \psi^{j}\left(p^{j}\right) \mp \frac{1}{2} \psi^{j}\left(p^{j}\right), \\
\gamma_{N}^{j, \pm} \mathrm{DL}_{k_{j}}^{j}\left(\phi^{j}\left(p^{j}\right)\right) & =-\mathrm{W}^{j} \phi^{j}\left(p^{j}\right),
\end{aligned}
$$

where $\mathrm{V}^{j}, \mathrm{~K}^{j}, \mathrm{~K}^{\prime j}, \mathrm{~W}^{j}$ are the single layer, double layer, adjoint double layer, and hypersingular boundary integral operators defined as follows

$\left[\bigvee^{j} \psi\left(p^{j}\right)\right](\mathbf{x})=\int_{\partial \Omega^{j}} G_{k_{j}}(\mathbf{x}, \mathbf{y}) \psi(\mathbf{y}) d \Gamma(\mathbf{y})$

for $\mathbf{x} \in \partial \Omega^{j}$,

$\left[\mathrm{K}^{j} \psi\left(p^{j}\right)\right](\mathbf{x})=\int_{\partial \Omega^{j}} \frac{\partial G_{k_{j}}(\mathbf{x}, \mathbf{y})}{\partial \mathbf{n}^{j}(\mathbf{y})} \psi(\mathbf{y}) d \Gamma(\mathbf{y})$

for $\mathbf{x} \in \partial \Omega^{j}$,

$\left[\mathrm{K}^{\prime j} \psi\left(p^{j}\right)\right](\mathbf{x})=$

$\frac{\partial}{\partial \mathbf{n}^{j}(\mathbf{x})} \int_{\partial \Omega^{j}} G_{k_{j}}(\mathbf{x}, \mathbf{y}) \psi(\mathbf{y}) d \Gamma(\mathbf{y})$, for $\mathbf{x} \in \partial \Omega^{j}$,

$\left[\mathbf{W}^{j} \psi\left(p^{j}\right)\right](\mathbf{x})=$

$-\frac{\partial}{\partial \mathbf{n}^{j}(\mathbf{x})} \int_{\partial \Omega^{j}} \frac{\partial G_{k_{j}}(\mathbf{x}, \mathbf{y})}{\partial \mathbf{n}^{j}(\mathbf{y})} \psi(\mathbf{y}) d \Gamma(\mathbf{y})$

Inserting the jump relations into Eq. (A.5) results in

$$
\begin{aligned}
& {\left[\begin{array}{c}
\mathrm{V}^{j} \psi^{j}\left(p^{j}\right)-\mathrm{K}^{j} \phi^{j}\left(p^{j}\right) \mp \frac{1}{2} \phi^{j}\left(p^{j}\right) \\
\mathrm{K}^{\prime j} \psi^{j}\left(p^{j}\right) \mp \frac{1}{2} \psi^{j}\left(p^{j}\right)+\mathrm{W}^{j} \phi^{j}\left(p^{j}\right)
\end{array}\right]=} \\
& {\left[\begin{array}{cc}
-\mathrm{K}^{j} \mp \frac{1}{2} \mathrm{Id} & \mathrm{V}^{j} \\
\mathrm{~W}^{j} & \mathrm{~K}^{j} \mp \frac{1}{2} \mathrm{Id}
\end{array}\right]\left[\begin{array}{l}
\phi^{j}\left(p^{j}\right) \\
\psi^{j}\left(p^{j}\right)
\end{array}\right],}
\end{aligned}
$$

which taking the identity matrix out gives the Calderón operator defined as follows

$\mathrm{A}_{k_{j}}^{j}:=\left[\begin{array}{cc}-\mathrm{K}^{j} & \mathrm{~V}^{j} \\ \mathrm{~W}^{j} & \mathrm{~K}^{\prime j}\end{array}\right]$,

thus, Eq. (A.5) becomes

$\gamma^{j,{ }^{\prime}} p^{j}=\left(\mp \frac{1}{2} \mathrm{ld}+\mathrm{A}_{k_{j}}^{j}\right)\left[\begin{array}{l}\phi^{j}\left(p^{j}\right) \\ \psi^{j}\left(p^{j}\right)\end{array}\right]$.

For the PMCHWT formulation, the interior jump potentials read

$\phi^{j}\left(p^{j}\right)=\gamma_{D}^{j,-} p^{j}$,

$\psi^{j}\left(p^{j}\right)=\gamma_{N}^{j,-} p^{j}$.

Taking Eqs. (A.13) and (A.14) into consideration, the Calderón representation of the interior Helmholtz problem in domain $\Omega^{j}$ reads.

$\gamma^{j,-} p^{j}=\left(\frac{1}{2} \mathrm{Id}+\mathrm{A}_{k_{j}}^{j}\right) \gamma^{j,-} p^{j}$

With regards the exterior domain, we define the total scattered field in $\Omega^{0}$ with $p_{s}$ and the total exterior pressure as $p_{\text {tot }}=p_{s}+p_{\text {inc }}$. Incorporating these definitions, the exterior jump potentials for PMCHWT formulation become $\phi^{0}\left(p^{0}\right)=-\gamma_{D}^{+} p_{\text {tot }}$ and $\psi^{0}\left(p^{0}\right)=-\gamma_{N}^{+} p_{\text {tot }}$, and $\gamma^{0,+} p=\gamma^{+} p_{s}$ where the Cauchy traces $\gamma^{+}$are evaluated at $\bigcup_{j=1}^{n} \partial \Omega^{j}$. Substituting these equations into Eq. (A.13) yields the Calderón formulation of the exterior Helmholtz problem as follows, one should note that the Calderón boundary operator is evaluated with the exterior wavenumber $k_{0}$,

$\gamma^{+} p_{s}=\left(\frac{1}{2} \mathrm{ld}-\mathrm{A}_{k_{0}}\right) \gamma^{+} p_{\mathrm{tot}}$

\section{References}

[1] P. Gélat, G. ter Haar, N. Saffari, The optimization of acoustic fields for ablative therapies of tumours in the upper abdomen, Phys. Med. Biol. 57 (2012) 8471-8497.
[2] P. Gélat, G. ter Haar, N. Saffari, A comparison of methods for focusing the field of a hifu array transducer through human ribs, Phys. Med. Biol. 59 (2014) 3139.

[3] P.V. Yuldashev, S.M. Shmeleva, S.A. Ilyin, O.A. Sapozhnikov, L.R. Gavrilov, V.A. Khokhlova, The role of acoustic nonlinearity in tissue heating behind a rib cage using a high-intensity focused ultrasound phased array, Phys. Med. Biol. 58 (2013) 
2537.

[4] J. Sun, K. Hynynen, Focusing of therapeutic ultrasound through a human skull: a numerical study, J. Acoust. Soc. Am. 104 (1998) 1705-1715.

[5] H.L. Liu, H. Chang, W.S. Chen, T.C. Shih, J.K. Hsiao, W.L. Lin, Feasibility of transrib focused ultrasound thermal ablation for liver tumors using a spherically curved $2 \mathrm{~d}$ array: a numerical study, Med. Phys. 34 (2007) 3436-3448.

[6] D. Pajek, K. Hynynen, The design of a focused ultrasound transducer array for the treatment of stroke: a simulation study, Phys. Med. Biol. 57 (2012) 4951.

[7] D. Pajek, K. Hynynen, The application of sparse arrays in high frequency transcranial focused ultrasound therapy: a simulation study, Med. Phys. 40 (2013) 122901.

[8] D. Liu, M.S. Adams, E.C. Burdette, C.J. Diederich, Transurethral high-intensity ultrasound for treatment of stress urinary incontinence (sui): simulation studies with patient-specific models, Int. J. Hyperth. 34 (2018) 1236-1247.

[9] U. Vyas, D. Christensen, Ultrasound beam propagation using the hybrid angular spectrum method, in: 2008 30th Annual International Conference of the IEEE Engineering in Medicine and Biology Society, IEEE, 2008, pp. 2526-2529.

[10] S.L. Johnson, D.A. Christensen, C.R. Dillon, A. Payne, Validation of hybrid angular spectrum acoustic and thermal modelling in phantoms, Int. J. Hyperth. 35 (2018) 578-590.

[11] C. Baron, J.F. Aubry, M. Tanter, S. Meairs, M. Fink, Simulation of intracranial acoustic fields in clinical trials of sonothrombolysis, Ultrasound Med. Biol. 35 (2009) 1148-1158.

[12] A. Kyriakou, E. Neufeld, B. Werner, G. Székely, N. Kuster, Full-wave acoustic and thermal modeling of transcranial ultrasound propagation and investigation of skullinduced aberration correction techniques: a feasibility study, J. Therapeutic Ultrasound 3 (2015) 11.

[13] M.K. Almekkaway, I.A. Shehata, E.S. Ebbini, Anatomical-based model for simulation of hifu-induced lesions in atherosclerotic plaques, Int. J. Hyperth. 31 (2015) 433-442.

[14] H.L. Liu, C.L. Hsu, S.M. Huang, Y.W. Hsi, Focal beam distortion and treatment planning for transrib focused ultrasound thermal therapy: a feasibility study using a two-dimensional ultrasound phased array, Med. Phys. 37 (2010) 848-860.

[15] V. Suomi, J. Jaros, B. Treeby, R.O. Cleveland, Full modeling of high-intensity focused ultrasound and thermal heating in the kidney using realistic patient models, IEEE Trans. Biomed. Eng. 65 (2018) 969-979.

[16] Y. Jing, T. Wang, G.T. Clement, A k-space method for moderately nonlinear wave propagation, IEEE Trans. Ultrason. Ferroelectr. Freq. Control 59 (2012) 1664-1673.

[17] S. Qiao, G. Shen, J. Bai, Y. Chen, Transcostal high-intensity focused ultrasound treatment using phased array with geometric correction, J. Acoust. Soc. Am. 134 (2013) 1503-1514.

[18] J. Gu, Y. Jing, Numerical modeling of ultrasound propagation in weakly heterogeneous media using a mixed-domain method, IEEE Trans. Ultrason. Ferroelectr. Freq. Control 65 (2018) 1258-1267.

[19] E. van 't Wout, P. Gélat, T. Betcke, S. Arridge, A fast boundary element method for the scattering analysis of high-intensity focused ultrasound, J. Acoust. Soc. Am. 138 (2015) 2726-2737.

[20] T. Betcke, E. van 't Wout, P. Gélat, Computationally Efficient Boundary Element Methods for High-Frequency Helmholtz Problems in Unbounded Domains, Springer International Publishing, 2017, pp. 215-243.

[21] S. Marburg, A pollution effect in the boundary element method for acoustic problems, J. Theoret. Comput. Acoust. 26 (2018) 1850018.

[22] J.L. Robertson, B.T. Cox, B.E. Treeby, Quantifying numerical errors in the simulation of transcranial ultrasound using pseudospectral methods, 2014 IEEE International Ultrasonics Symposium, 2014, pp. 2000-2003, , https://doi.org/10. 1109/ULTSYM.2014.0498.

[23] F.A. Duck, Physical Properties of Tissues: A Comprehensive Reference Book, Academic Press, 2013.

[24] R. Ritchie, J. Collin, C. Coussios, T. Leslie, Attenuation and de-focusing during highintensity focused ultrasound therapy through peri-nephric fat, Ultrasound Med. Biol. 39 (2013) 1785-1793.

[25] D. Cranston, A review of high intensity focused ultrasound in relation to the treatment of renal tumours and other malignancies, Ultrason. Sonochem. 27 (2015) 654-658.

[26] P. Ramaekers, M. Ries, C.T. Moonen, M. de Greef, Improved intercostal hifu ablation using a phased array transducer based on fermat's spiral and voronoi tessellation: a numerical evaluation, Med. Phys. 44 (2017) 1071-1088.

[27] C. Cheng, A. Seybert, T. Wu, A multidomain boundary element solution for silencer and muffler performance prediction, J. Sound Vib. 151 (1991) 119-129.

[28] A. Medeiros, T. Macarios, G. Azevedo, B. Gardner, Predicting Transmission Loss of Mufflers with Perforated Tube Elements using the Boundary Element Method. Technical Report. SAE Technical Paper, 2014.

[29] S. Huang, Y. Liu, A new simple multidomain fast multipole boundary element method, Comput. Mech. 58 (2016) 533-548.

[30] D. Brunner, M. Junge, P. Rapp, M. Bebendorf, L. Gaul, Comparison of the fast multipole method with hierarchical matrices for the helmholtz-bem, Comput. Model. Eng. Sci. (CMES) 58 (2010) 131-160.

[31] H. Wu, Y. Liu, W. Jiang, A fast multipole boundary element method for 3d multidomain acoustic scattering problems based on the burton-miller formulation, Eng Anal. Boundary Elem. 36 (2012) 779-788.

[32] W. Śmigaj, T. Betcke, S. Arridge, J. Phillips, M. Schweiger, Solving boundary in tegral problems with bem ++ , ACM Trans. Math. Softw. 41 (2015) 6:1-6:40, https://doi.org/10.1145/2590830.

[33] M.F. Hamilton, D.T. Blackstock, et al., Nonlinear Acoust. 237 (1998),

[34] R. Hiptmair, Operator preconditioning, Comput. Math. Appl. 52 (2006) 699-706.

[35] R.C. Kirby, From functional analysis to iterative methods, SIAM Rev. 52 (2010) 269-293.

[36] X. Antoine, M. Darbas, Integral Equations and Iterative Schemes for Acoustic Scattering Problems, in: F. Magoulès (ed.), Numerical Methods for Acoustics Problems, Saxe-Coburg Editors, 2016.

[37] M. Gossye, M. Huynen, D. Vande Ginste, D. De Zutter, H. Rogier, A calderón preconditioner for high dielectric contrast media, IEEE Trans. Antennas Propag. 66 (2018) 808-818.

[38] Xavier Antoine, Marion Darbas, Generalized combined field integral equations for the iterative solution of the three-dimensional helmholtz equation, ESAIM: M2AN 41 (2007) 147-167. doi:10.1051/m2an:2007009.

[39] M. Darbas, E. Darrigrand, Y. Lafranche, Combining analytic preconditioner and fast multipole method for the 3-d helmholtz equation, J. Comput. Phys. 236 (2013) 289-316, https://doi.org/10.1016/j.jcp.2012.10.059.

[40] X. Antoine, H. Barucq, A. Bendali, Bayliss-Turkel-like radiation conditions on surfaces of arbitrary shape, J. Math. Anal. Appl. 229 (1999) 184-211.

[41] X. Antoine, Advances in the on-surface radiation condition method: theory, numerics and applications, in: F. Magoulès (Ed.), Computational Methods for Acoustics Problems, Saxe-Coburg Publications, 2008, pp. 169-194.

[42] X. Antoine, Y. Boubendir, An integral preconditioner for solving the two-dimensional scattering transmission problem using integral equations, Int. J. Comput. Math. 85 (2008) 1473-1490.

[43] V.C. Anderson, Sound scattering from a fluid sphere, J. Acoust. Soc. Am. 22 (1950) 426-431.

[44] P.M.P.M. Morse, Theoretical acoustics. International series in pure and applied physics, McGraw Hill, New York, 1968.

[45] P. Hasgall, F. Di Gennaro, C. Baumgartner, et al., IT'IS database for thermal and electromagnetic parameters of biological tissues v4.0, 2018. doi:10.13099/ VIP21000-04-0.

[46] F.A. Duck, Physical Properties of Tissue: A Comprehensive Reference Book, Francis A. Duck. Academic Press, London, 1990.

[47] B.D. de Senneville, C. Moonen, M. Ries, Mri-guided hifu methods for the ablation of liver and renal cancers, Therapeutic Ultrasound, Springer, 2016, pp. 43-63.

[48] R. Perz, J. Toczyski, D. Subit, Variation in the human ribs geometrical properties and mechanical response based on $\mathrm{x}$-ray computed tomography images resolution, J. Mech. Behav. Biomed. Mater. 41 (2015) 292-301.

[49] S.A. Holcombe, E. Hwang, B.A. Derstine, S.C. Wang, Measuring rib cortical bone thickness and cross section from ct, Med. Image Anal. 49 (2018) 27-34. 